\title{
An Evaluation of Classification Techniques for Depression, Anxiety and Stress Assessment
}

\author{
${ }^{1}$ S. T. Arokkiya Mary, ${ }^{2}$ Dr. L. Jabasheela \\ ${ }^{1}$ Research scholar, Research and Development Centre, Bharathiar University, Coimbatore, India \\ ${ }^{2}$ Professor, Panimalar Engineering College, Chennai, India \\ arokya25@yahoo.co.in, ljsheela@gmail.com
}

\begin{abstract}
In the past decades, Depression, Anxiety and Stress (DAS) became a serious health issue in the society over all parts of the world. It can be measured by Depression, Anxiety and Stress Scale (DASS) which consists of a set of questionnaires. Several techniques are proposed to validate the DASS level and to identify the levels of severity. The goal of this paper is to predict the DAS level using 5 different classifiers namely Logistic Regression (LR), Multilayer Perceptron (MLP), J48, Reduces Error Pruning (REP) Tree and Classification and Regression Trees (CART) algorithm. The major intention of this study is to compare the performance of these 5 classifiers based on classification accuracy, kappa value, precision, recall, F-score and ROC. We assessed the classifiers of the DASS-21 version suing the samples collected from 600 students from Puducherry, India. For experimentation, WEKA is used as a simulation tool and the results reveal that the MLP achieves better performance when compared to other classifiers. MLP attains the classification accuracy of $90.33 \%, 92 \%$ and $90.33 \%$ for Depression, Anxiety and Stress dataset respectively.
\end{abstract}

Keywords : DAS,REP,CART,MLP.

\section{INTRODUCTION}

In the year of 2011, World Health Organization (WHO) stated that the mental health is a state of well-being where each person recognizes their individual capabilities, can handle the stress in life, and also contributes their effort to the society. A study conducted in [Indus 1] revealed that around 450 million people suffer from a kind of mental illness [1]. In the state of being mental illness, DAS plays a major role and $13-14 \%$ of people from world's population are affected by DAS [2]. Another study conducted in India [3] expresses that $2 \%$ of Indians experiences mental illness and $6-10 \%$ of people have emotional disorder. The occurrence of depression rate among Indians is increased from 5.8 to 9.5 in a year. The occurrence of anxiety rate among Indians is 16.5 and stress rate is found to be 9.5. [4]. It is also observed that major of the stress problem occurs due to working environment [5]. It is already known that the working environment is a complex structure. A person can gain the positive effect in work place like secured job, convenient time or exposure [6]. At the same time, negative impacts are also present due to the lack of profit, unpleasant surrounding, less production and so on [7].

Depression is a general psychological illness which represents the depressed state of mind, disinterest, guilty feeling, lack of sleep or hunger and less concentration. Generally, depression is a state which significantly affects the person's potential to do regular activities and also results to maximized risk of self-injury or suicide [8]. WHO defines that $5 \%$ of people suffers from depression in the last year. The symptoms usually start at the younger age itself[9]. The occurrence rate of depression in young people is found to be higher. In [10], a study indicates that the frequency of occurrence of the depression rate is $15.6 \%$ in undergraduate students was $15.6 \%$, while the more recent one found 9-13\% [11]. On the other hand, presently, numerous students undergone to depressed state which cannot be identified [12]. Once the students in depressed state are recognized and proper medicated are given, dangerous effects of depression and suicide can be avoided [13].

There are several factors which influence DAS and some of the reasons are personal problems, illness, pregnancy, academic progress, social media, disabled students, etc. the earlier identification of these problems are necessary to provide treatment and decreasing the disability in environment [19].Different measuring scales are present to measure the levels of DAS and some of them include Beck Depression Inventory (BDI), the Beck Anxiety Inventory (BAI), the Hospital Anxiety and Depression Scale (HADS) [20], the Center for Epidemiological Studies Depression (CES-D), and the Depression Anxiety Stress Scales (DASS). DASS is the most commonly used measuring scale and it has two versions namely DASS-21 and DASS-42.

\section{RELATED WORK}

In this section, we review the existing approaches to analyze DASS in several aspects. The existing methods are explained with its objectives, methodology, statistical analysis, benefits and limitations.

In [21], the authors performed a study to investigate the satisfactoriness and verification of the suggestion of providing internet-delivered treatment DAS for university students. The outcome of the study shows that he DAS level is decreased among students who undergone the proposed learning 4 times in a month. [22]performed an experiment to investigate the sate of DAS on high school girl students in Saudi Arabia using DASS-42. Among the samples ( $\mathrm{N}=545)$, only $26 \%$ of girls are not having DAS and half of the girls suffers from atleast two 
illness in DAS. The results depict the significance of

\begin{tabular}{|c|c|}
\hline $1(\mathrm{~s})$ & I found it hard to wind down \\
\hline 2 (a) & I was aware of dryness of my mouth \\
\hline $3(d)$ & I couldn't seem to experience any positive feeling at all \\
\hline 4 (a) & $\begin{array}{l}\text { I experienced breathing difficulty (e.g. excessively rapid breathing, } \\
\text { breathlessness in the absence of physical exertion) }\end{array}$ \\
\hline 5 (d) & I found it difficult to work up the initiative to do things \\
\hline $6(s)$ & I tended to over-react to situations \\
\hline 7 (a) & I experienced trembling (e.g. in the hands) \\
\hline $8(s)$ & I felt that I was using a lot of nervous energy \\
\hline 9 (a) & $\begin{array}{l}\text { I was worried about situations in which I might panic and make a fool } \\
\text { of myself }\end{array}$ \\
\hline 10 (d) & I felt that I had nothing to look forward to \\
\hline $11(s)$ & I found myself getting agitated \\
\hline $12(s)$ & I found it difficult to relax \\
\hline 13 (d) & I felt down-hearted and blue \\
\hline $14(s)$ & $\begin{array}{l}\text { I was intolerant of anything that kept me from getting on with what I } \\
\text { was doing }\end{array}$ \\
\hline 15 (a) & I felt I was close to panic \\
\hline 16 (d) & I was unable to become enthusiastic about anything \\
\hline 17 (d) & I felt I wasn't worth much as a person \\
\hline $18(s)$ & I felt that I was rather touchy \\
\hline 19 (a) & $\begin{array}{l}\text { I was aware of the action of my heart in the absence of physical } \\
\text { exertion (e.g. sense of heart rate increase, heart missing a beat) }\end{array}$ \\
\hline 20 (a) & I felt scared without any good reason \\
\hline 21 (d) & I felt that life was meaningless \\
\hline
\end{tabular}

Fig. 1. DASS-21 measure

appointing primary care physicians to guard girls, validate and heal mental illness. When a person suffers from heart failure, there is a greater chance of having DAS and the probability of death is increased. [23] examines the interconnection between spiritual health and DAS in the patients suffers from heart problems. A sample $(\mathrm{N}=150)$ is collected from Ardabil hospitals in the year of 2014. The outcome of this study expresses that the increase in spiritual health automatically decreases the level of DAS levels in heart failure patients. It suggests the necessity of forming medical communities to give mandatory spiritual healthcare.

A survey reported that the presence of a relationship between video game addiction and mental illness, but few research have involved in it. There is an uncertainty to differentiate the highly involved level of video games from addiction. [24]conducted an investigation to determine the intermediate responsibility to handle the measure of addicting to video game and mental illness. A survey (samples $\mathrm{N}=552$ ) is conducted thorough internet using CAES scale, DASS-21 and BACQ. The outcome represents there is a strong relation between them the higher involvement in video games can lead to higher risk of addiction.

[28] performed an experiment to inspect the DAS level on medical undergraduate students by DASS-42. DASS 42 is

used to obtain information about society based and personal habits (cigarette smoke, alcohol, academic performance). The results show that approximately half of the students suffer from DAS and the respective percentage are $51 \%, 70 \%$ and $60 \%$. It is also noted that girl's students are having higher level of DAS than male students. It is also recommended to organize counseling to the students on a regular basis. Another study [29] is carried out to observe the DAS level on 143 first year medical students from India. 143 medical students from India were taken as sample and collected data using DASS-42. The results indicate the respectively percentages are $46.85 \%$, $74.12 \%$ and $52.44 \%$. An Italian version of DASS-21 is developed in [30] to detect the levels of DAS accurately. A sample of 417 participants and two clinical groups (32 depressive patients and 25 anxious patients) has undergone the Italian version of DASS-21 in addition to several measures of psychopathology.

\section{DASS 21 MEASURE}

Several measuring scales are present for the purpose of computing the level of DAS. DASS is the widely employed measuring to identify the warning sign of DAS in the physical world. DASS tool comprises of 3 sub-scales for DAS. And they fall into two categories namely DASS-21 and DASS-42.

DASS 21 is the former version holds 21 items and each 7 items belongs to a sub-scale as shown in Fig. 1. DAS-42 includes the entire list of 42 items. The scores of DAS are calculated by the total score of the individual relevant items

TABLE I

VARIOUS LEVELS OF DAS SCORES

\begin{tabular}{|l|c|c|c|}
\hline & Depression & Anxiety & Stress \\
\hline Normal & $0-9$ & $0-7$ & $0-14$ \\
\hline Mild & $10-13$ & $8-9$ & $15-18$ \\
\hline Moderate & $14-20$ & $10-14$ & $19-25$ \\
\hline Severe & $21-27$ & $15-19$ & $26-33$ \\
\hline Extremely Severe & $28+$ & $20+$ & $34+$ \\
\hline
\end{tabular}

and the levels are categorized using the DAS score. The ranges of DAS score with the corresponding levels are given in Table 1 . The DASS-21 is listed below with their rating criteria. Recommended cut-off scores for conventional severity levels are also mentioned below.

\section{A. Logistic Regression}

Logistic regression [35] is a conventional statistical analysis method which employs the logits (score) to identify the target class. It is a type of linear classifies which determines the relation between categorical dependent variable and one or more independent variables by calculating probabilities. It utilizes a black box testing termed as Softmax function. Logistic distribution creates the basis of the logit model with 
its distribution function as equated in Eq. (1).

$$
F\left(X_{i} \beta\right)=\frac{\exp \left(X_{i} \beta\right)}{1+\exp \left(X_{i} \beta\right)}
$$

The density function is given in Eq. (2).

$$
\frac{\exp \left(X_{i} \beta\right)}{\left[1+\exp \left(X_{i} \beta\right)\right]^{2}}
$$

\section{B. Multilayer Perceptron}

MLP [36] is the famous neural network based method which activated through the process of loading the input layer with the input vector and propagating actions in a feed forward manner via weighted connections in the complete network. For an input $w_{k}$, the state of $i^{t h}$ neuron $\left(s_{i}\right)$ is computed as

$$
s_{i}=f\left(w_{i, 0}+\sum_{j \in P_{i}} w_{i, j} \times s_{j}\right)
$$

where $\boldsymbol{f}$ is the activation function, $\boldsymbol{P}_{\boldsymbol{i}}$ is the group of nodes reaching node $\boldsymbol{i}, \boldsymbol{w}_{i, j}$ is the weight of the connection between node $\boldsymbol{i}$ and $\boldsymbol{j}$. MLP employs an iterative function for learning process which begins from random weights. A training algorithm is also utilized to manage the weights to an intended target values. The training will be stopped only when the error slope comes to zero.

\section{Classification and Regression Trees (CART)}

CART is a machine-learning approach to design the prediction models from data. The models are developed by iteratively dividing the data space and placing an easy prediction model for every division. Consequently, the resultant divisions can be pictorially represented as a decision tree. Classification trees are developed for interdependent variables that uses a countable number of unordered values, with prediction error computed based on misclassification cost. Next, Regression trees are for dependent variables which include continuous or ordered discrete values, with prediction error commonly quantified by the squared difference between the observed and predicted values. CART forms the basis of many popular algorithms such as bagged decision trees, random forest and boosted decision trees. The three major components in CART are: rules to split data, stopping rules and the prediction of the target variable.

\section{D. $\quad J 48$}

J48 is an extended version of ID3 classifier [37]. The extra characteristic of J48 is the consideration of missing values, pruning DT, derivation of rules and so on. In WEKA, it is an open source Java implementation of the C4.5 algorithm. It controls both continuous and discrete variables. A threshold value is computed to handle continuous attributes. The value partition the data list into attributed falling lesser than threshold value and attributes falling higher or equal to the threshold value. It also manages missing values in the training process. When the tree is completely built, pruning process will takes place.

\section{E. Reduced Error Pruning (REP) Tree Classifier}

REP tree is the faster DT algorithm which works on the basis of calculating information gain with entropy and reducing the error appears from variance [38]. REP Tree is applied to regression tree logic and creates many trees in various iterations. Next, it chooses the best tree from all other spawned trees. It builds the DT based on variance and information gain. In addition, it prunes the tree through reduced-error pruning with back fitting method. The numeric variables are sorted in the initialization level of the model preparation. REP Tree manages the missing values by dividing the respective instances to pieces.

\section{Performance Evaluation}

In this section, the samples collected from 600 college students from Puducherry, India using DASS-21 measure to assess the level of DAS among them. The dataset consists of a total of 600 instances. For experimentation, WEKA is used as a simulation tool. The same dataset is applied to all the above mentioned 5 classifiers. The results are analyzed based on some metrics such as classification accuracy, kappa value, precision, recall, F-score and ROC. The experimental results of various classifiers to inspect DAS are tabulated in Table 2, Table 3 and Table 4 respectively. The comparison results are also pictorially represented in Fig 2, Fig. 3 and Fig. 4 respectively. From Table 2, it is clear that CART attains the poor performance for Depression dataset with an $80 \%$ accuracy whereas LP achieves better accuracy of $90.33 \%$ but lesser than MLP. Table 3 depicts that REP Tree fails to achieve better accuracy than other methods on Anxiety dataset. From Table 4, it is shown that CART produces poor results than the compared methods. MLP attains the classification accuracy of $90.33 \%, 92 \%$ and $90.33 \%$ for Depression, Anxiety and Stress dataset respectively. The above discussion reveals that MLP is the best classifier to identify the levels of DAS using DASS-21. 
TABLE II

COMPARISON OF DEPRESSION DATASET WITH VARIOUS CLASSIFIERS

\begin{tabular}{|c|c|c|c|c|c|c|}
\hline Classifier & Accuracy & Kappa & Precision & Recall & F-score & ROC \\
\hline \hline LR & 90.33 & 72.00 & 90.90 & 90.30 & 90.50 & 47.6 \\
\hline MLP & 94.16 & 82.83 & 94.00 & 94.20 & 94.00 & 95.10 \\
\hline J48 & 82.16 & 43.00 & 80.10 & 82.20 & 81.00 & 84.10 \\
\hline RepTree & 81.50 & 34.08 & 76.30 & 81.50 & 78.20 & 82.00 \\
\hline CART & 80.00 & 33.10 & 75.00 & 80.00 & 77.89 & 81.23 \\
\hline
\end{tabular}

TABLE III

COMPARISON OF ANXIETY DATASET WITH VARIOUS CLASSIFIERS

\begin{tabular}{|c|c|c|c|c|c|c|}
\hline Classifier & Accuracy & Kappa & Precision & Recall & F-score & ROC \\
\hline \hline LR & 92.00 & 73.44 & 93.40 & 92.00 & 92.60 & 54.60 \\
\hline MLP & 99.50 & 84.71 & 95.40 & 95.50 & 95.40 & 97.20 \\
\hline J48 & 86.33 & 47.10 & 84.00 & 86.30 & 84.90 & 85.40 \\
\hline RepTree & 85.16 & 38.20 & 81.00 & 85.20 & 82.50 & 78.20 \\
\hline CART & 88.66 & 51.25 & 86.10 & 88.70 & 86.30 & 88.10 \\
\hline
\end{tabular}

TABLE IV

COMPARISON OF STRESS DATASET WITH VARIOUS CLASSIFIERS

\begin{tabular}{|c|c|c|c|c|c|c|}
\hline Classifier & Accuracy & Kappa & Precision & Recall & F-score & ROC \\
\hline \hline LR & 90.33 & 72.00 & 90.90 & 90.30 & 90.50 & 47.6 \\
\hline MLP & 94.16 & 82.83 & 94.00 & 94.20 & 94.00 & 95.10 \\
\hline J48 & 82.16 & 43.00 & 80.10 & 82.20 & 81.00 & 84.10 \\
\hline RepTree & 81.50 & 34.08 & 76.30 & 81.50 & 78.20 & 82.00 \\
\hline CART & 80.00 & 33.10 & 75.00 & 80.00 & 77.89 & 81.23 \\
\hline
\end{tabular}

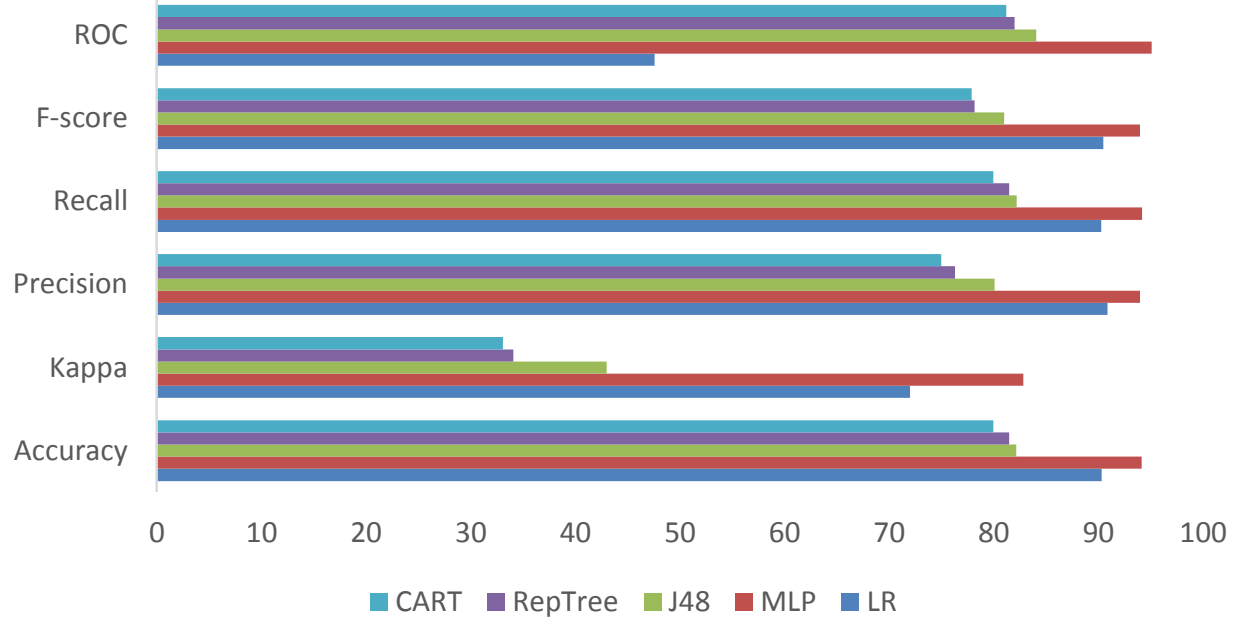

Figure.2. Comparison on Depression dataset with various classifiers 


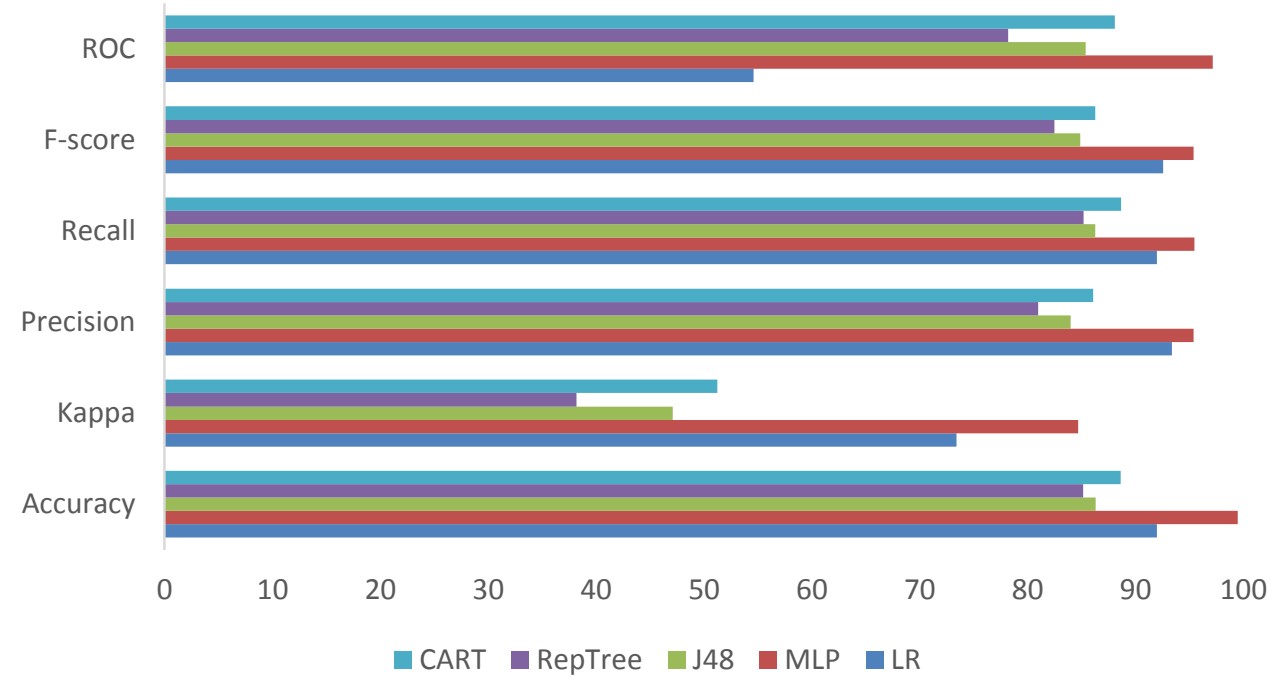

Figure 3. Comparison on Anxiety dataset with various classifiers

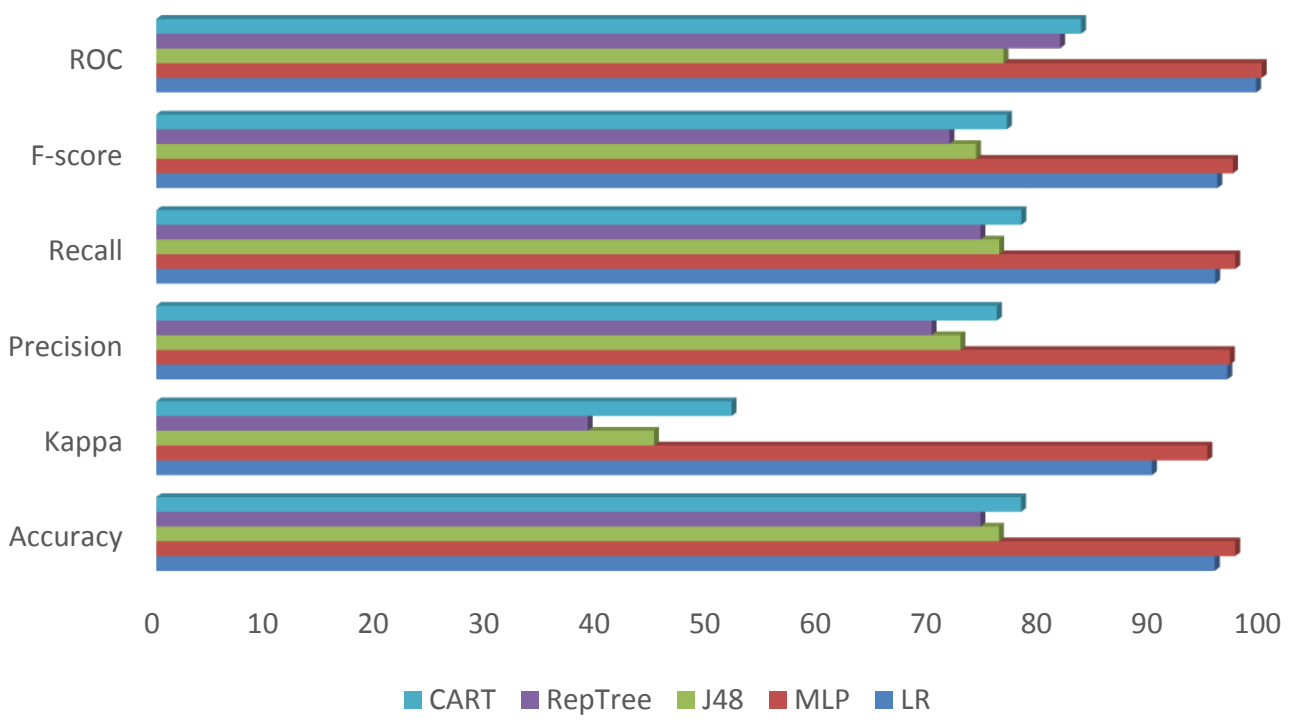

Figure. 4. Comparison on Stress dataset with various classifiers

\section{CONCLUSION}

In this paper, 5 different classifiers namely Logistic Regression (LR), Multilayer Perceptron (MLP), J48, REP Tree and CART algorithm are applied to assess the level of DAS using DASS-21. The major intention of this study is to compare the performance of these 5 classifiers based on classification accuracy, kappa value, precision, recall, F-score and ROC. We assessed the classifiers of the DASS-21 version suing the samples collected from 600 students from Puducherry, India. For experimentation, WEKA is used as a simulation tool and the results reveal that the LR achieves better performance when compared to other classifiers. LR attains the classification accuracy of $90.33 \%, 92 \%$ and $90.33 \%$ for Depression, Anxiety and Stress dataset respectively.

\section{REFERENCES}

[1] R.S. Murthy, A. Haden, B.Campanini, editors.Mental Health: New Understanding, New Hope. World Health Report, Geneva. pp.9, 2001.

[2] Mental Health Foundation, UK: Economic burden of Mental Health Illness cannot be tackled without Research Investment. [Internet] Available from: http://www.mentalhealth.org.uk/ content/assets/PDF/campaigns/MHF-Business-case-for-MHresearchNov2010.pdf [Last cited on 2010 Nov].

[3] WHO Statistics, India, World Health Organisation, 2010,Geneva. [Internet] Available from: http://www.who. int/countries/ind/en/[Last cited on 2010 Sept].

[4] H.C. Ganguli HC., "Epidemiological finding on prevalence of mental disorders in India," Indian J Psychiatry vol. 42, no. : 14, 2000.

[5] Mishra, S. Mehta, N.D. Sinha, S.K. Shukla, N. Ahmed and Kawatra A., "Evaluation of workplace stress in health university workers: A study from rural India," Indian J Community Med., vol.36, pp.39-44, 2011.

[6] Harnois and P. Gabriel., "Mental Health: Issues, Impact and Practices. Geneva:World Health Organisation" pp.5-7, 2000.

[7] T. Rajgopal, "Mental well-being at the workplace." Indian J Occup Environ Med., vol.14, pp. 63-5, 2010. 
[8] L. White, C. Klinner and S. Carter, "Consumer perspectives of the Australian Home Medicines Review Program: benefits and barriers. Res SocAdm Pharm., vol. 8, no. 1, pp. 4-16, 2012

[9] World Federation for Mental Health (2012) Depression: a global crisis. In: World Health Organization. Accessed 4 Dec 2017, Available via: http://www.who.int/mental_health/management/depression/wfmh_paper _depression_wmhd_2012.pdf?ua=1.

[10] Eisenberg, S.E.Gollust, E. Golberstein and J.L.Hefne, "Prevalence and correlates of depression, anxiety, and suicidality among university students. Am J Orthopsychiatry, vol. 77, no. 4, pp. 534-542, 2007.

[11] Richards and A.Sanabria, "Point-prevalence of depression and associated risk factors," J Psychol., vol. 148, no. 3, pp.305-326, 2014

[12] Ovuga, J. Boardman and D.Wasserman, "The response inventory for stressful life events (RISLE) II: validation of the 36-item version," Afr Health Sci., vol. 5, no. 2, pp.145-151, 2005.

[13] P.S.Wang, M. Angermeyer, G. Borges, R.Bruffaerts, W. Tat Chiu and G. Girolamo, "Delay and failure in treatment seeking after first onset of mental disorders in the World Health Organization's World Mental Health Survey Initiative," World Psychiatry, vol. 6, no. 3, pp.177-185, 2007

[14] DP and L. Y:, Form and frequency of mental disorders across centres. In Mental Illness in General Health Care. NY: John Wiley \& Sons, 1997.

[15] S. L. Currie, P. J. Mcgrath, and V. Day, "Computers in Human Behavior Development and usability of an online CBT program for symptoms of moderate depression , anxiety , and stress in post-secondary students," Comput. Human Behav., vol. 26, no. 6, pp. 1419-1426, 2010

[16] P. Frazier et al., "Acceptability and proof of concept of internetdelivered treatment for depression, anxiety, and stress in university students : protocol for an open feasibility trial," Pilot Feasibility Stud., pp. 1-9, 2016.

[17] S. Arabia, "Restructuring and Restructuring of the International Court of Justice", 42- "Declaration of Principles and Principles for the Reform of the Temple," vol. 9, no. June, pp. 140-147, 2009.

[18] M. Safavi, N. Oladrostam, M. Fesharaki, and Y. Fatahi, "An Investigation of the Relationship between Spiritual Health and Depression , Anxiety, and Stress in Patients with Heart Failure," vol. 3, no. 2, pp. 2-7, 2016.

[19] D. Loton and E. Borkoles, "Running head: VIDEO GAME ADDICTION, ENGAGEMENT AND COPING.”,2015.
[20] Kaur, G. H. Tee, S. Ariaratnam, and A. S. Krishnapillai, "Depression, anxiety and stress symptoms among diabetics in Malaysia: a cross sectional study in an urban primary care setting," 2013.

[21] S. Rao and N. Ramesh, "Depression, anxiety and stress levels in industrial workers: A pilot study in Bangalore, India," Ind. Psychiatry J., vol. 24, no. 1, pp. 23-28, 2015.

[22] K. Singh, M. Junnarkar, and S. Sharma, "Anxiety, stress, depression, and psychosocial functioning of Indian adolescents," vol. 57, no. 4, 2017.

[23] S. Iqbal, S. Gupta, and E. Venkatarao, "stress, anxiety \& depression among medical undergraduate students \& their socio-demographic correlates," no. March, pp. 354-357, 2015.

[24] K. K. Patnaik, C. M. Samuel, G. B. Pathrudu, Y. V. A. Ramlakshmi, F. Bahmed, and A. V. P. Bodhe, "Depression, anxiety \& stress among first year medical students : A cross sectional study .," vol. 14, no. 10, pp. $25-28,2015$.

[25] Bottesi, M. Ghisi, G. Altoè, E. Conforti, G. Melli, and C. Sica, "ScienceDirect The Italian version of the Depression Anxiety Stress Scales-21: Factor structure and psychometric properties on community and clinical samples," Compr. Psychiatry, 2015.

[26] M. Gamon, S. Counts, and E. Horvitz, "Predicting Depression via Social Media," vol. 2,2013.

[27] D. Kunwar, A. Risal, and S. Koirala, "Study of Depression , Anxiety and Stress among the Medical Students in two Medical Colleges of Nepal," vol. 53, no. 1, pp. 22-26, 2016.

[28] O. Journal, E. Sciences, and A. Akin, "Internet Addiction and Depression, Anxiety and Stress," vol. 3, no. 1, pp. 138-148, 2011.

[29] B. Ajilchi and V. Nejati, "Research Paper: Executive Functions in Students With Depression, Anxiety, and Stress Symptoms," vol. 8, no. 3, pp. 223-232, 2017.

[30] S.S. Haykin, "Neural networks and learning machines," Prentice-Hall, Englewood Cliffs, 2009

B. Back, T. Laitinen, K. Sere, "Neural networks and genetic algorithms for bankruptcy prediction," Expert Systems with Applications, vol. 11, pp.407-413, 2013. 